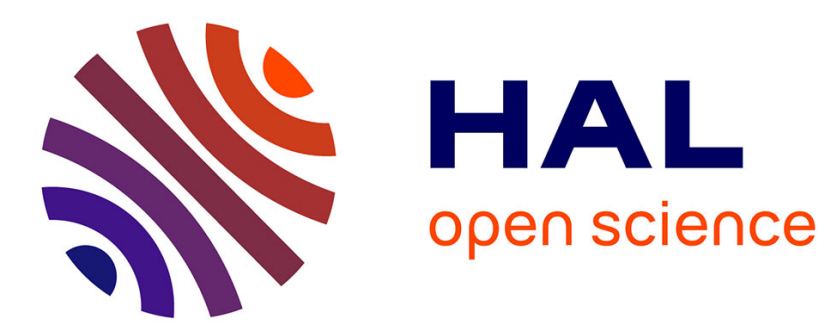

\title{
Laissés-pour-compte et hommes au rebut en démocratie: Kafka et Melville
}

Frédérique Leichter-Flack

\section{To cite this version:}

Frédérique Leichter-Flack. Laissés-pour-compte et hommes au rebut en démocratie: Kafka et Melville. Revue de Litterature Comparee, 2009, 329, pp.41-54. 10.3917/rlc.329.0041 . hal-01984631

\section{HAL Id: hal-01984631 \\ https://hal.parisnanterre.fr/hal-01984631}

Submitted on 17 Jan 2019

HAL is a multi-disciplinary open access archive for the deposit and dissemination of scientific research documents, whether they are published or not. The documents may come from teaching and research institutions in France or abroad, or from public or private research centers.
L'archive ouverte pluridisciplinaire HAL, est destinée au dépôt et à la diffusion de documents scientifiques de niveau recherche, publiés ou non, émanant des établissements d'enseignement et de recherche français ou étrangers, des laboratoires publics ou privés. 


\title{
Laissés-pour-compte et hommes au rebut en démocratie : Kafka et Melville
}

\author{
Un homme à la mer! \\ Qu'importe! le navire ne s'arrête pas. Le \\ vent souffle, ce sombre navire-là a une \\ route qu'il est forcé de continuer. Il passe. \\ [...] Il était là tout à l'heure, il était de l'équi- \\ page, il allait et venait sur le pont avec les \\ autres, il avait sa part de respiration et de \\ soleil, il était un vivant. Maintenant, que \\ s'est-il donc passé ? Il a glissé, il est tombé, \\ c'est fini. \\ [...] Ô marche implacable des sociétés \\ humaines! \\ [...] La mer, c'est l'immense misère. ${ }^{1}$
}

L'image de l'homme à la mer, que le navire Société abandonne à son sort sans même entendre sa détresse, semble aujourd'hui une métaphore convenue. Mais son usage médiatique contemporain ne l'entend pas dans le sens que le roman hugolien lui donnait. «La mer, c'est l'inexorable nuit sociale où la pénalité jette ses damnés »: le Hugo des Misérables dénonçait le passeport jaune qui condamnait l'ancien forçat Jean Valjean à coucher dehors tandis que toutes les portes demeuraient fermées devant lui. Pour lui, la machine à fabriquer des parias, c'était la Loi, une sorte de monstre rigoureux, inflexible, inaccessible à tout sentiment humain, et parfois injuste, qui n'accorde pas de seconde chance : la stigmatisation pénale est définitive, et la noyade certaine, à moins d'un miracle que la fiction n'accorde à Jean Valjean que pour en dénoncer la rareté. À cette problématique datée ${ }^{2}$ a succédé, au travers de la même métaphore, le phénomène de l'exclusion sociale. Dans le contexte politique de nos démocraties libérales, la question centrale

1. Victor Hugo, Les Misérables, Paris, Laffont, coll. «Bouquins », Ire partie, Livre II, chapitre 8, p. 77-78.

2. Qui conserve cependant son actualité, comme en témoignent, entre autres, les dysfonctionnements des processus de réinsertion à la sortie de prison dans la France d'aujourd'hui. 
n'est plus tant celle de la Loi, que celle de l'utilité sociale. La vague qui emporte le malheureux hors du pont, ce n'est plus la pénalité, c'est le libre jeu du marché, et le paria moderne, non plus (seulement) celui que la Loi a attrapé au collet, mais celui que la misère, le chômage, la disqualification professionnelle, ont sorti du terrain de jeu social : laissés-pour-compte et hommes au rebut, tous ceux qui n'ont pas assez d'équilibre pour tenir sur le pont au milieu d'une mer déchaînée, tous ceux qui ne trouvent pas leur place, ou ne savent pas la garder, tous ceux qui ne savent pas jouer le jeu.

Relus ensemble sur la base d'un rapprochement comparatiste fondé sur cette intuition de lecture, deux textes bien connus - Bartleby le scribe de Melville et « le Soutier », premier chapitre du premier roman de Kafka, Amerika ou le Disparu -, nous montreront comment la littérature se charge de cette problématique. Sur fond d'un scénario d'exclusion kafkaïen, la superposition de ces deux scènes politiques américaines produit des effets de sens qui articulent de façon exemplaire l'enjeu éthique et l'enjeu politique.

Bartleby et «le Soutier $»^{3}$ : comment rapprocher ces deux textes qui s'ignorent? Dans les deux cas, il est question d'un pauvre homme qu'un autre, mieux inséré socialement, tente de sauver, puis abandonne à son sort. Dans Amerika ou le Disparu, c'est un soutier anonyme, victime de discrimination de la part de son supérieur hiérarchique et sur le point d'être débarqué du navire où il travaille, qui se trouve un temps défendu - sans succès - par le jeune Karl Rossmann. Chez Melville, c'est un ancien scribe totalement démuni, Bartleby, qui persiste à occuper les bureaux de son patron et parvient, un temps lui aussi, à s'y faire tolérer. L'issue, explicite chez Melville, implicite chez Kafka, est en réalité comparable. Bartleby, on s'en souvient, meurt solitaire dans la prison où il est jeté quand la tolérance à son égard prend fin. Quand au soutier du roman de Kafka, on peut le supposer, à la fin de l'épisode, renvoyé à l'anonymat de la foule misérable des limbes du port de New York; du reste, le récit l'abandonne à son sort sans même conclure à son sujet, et tandis qu'il s'éloigne du navire, en chaloupe, avec l'oncle sénateur qu'il vient de se découvrir, Karl Rossmann se prend à penser que « tout se passait vraiment comme s'il n'y avait plus eu de soutier » $(A 51)^{4}$. Chacun de ces deux textes orchestre donc un scénario d'exclusion sociale: un homme tombe à la mer, s'y maintient à la surface tout le temps qu'un autre lui tend la main, puis s'y noie, quand la main secourable lui est retirée.

3. On se référera à ces deux textes à partir des éditions suivantes : Melville, Bartleby le scribe/Bartleby the scrivener, trad. Pierre Leyris, Paris, Gallimard, coll. « folio bilingue », n¹15, 2003, et Kafka, Amerika ou le Disparu, chapitre 1 《Le soutier », trad. Bernard Lortholary, Paris, Garnier Flammarion, 1988. Les numéros de pages mentionnés ci-après renvoient à ces deux éditions, respectivement $B$ et $A$. Les citations en version originale de Bartleby renverront à cette même édition $(B)$. Les citations en version originale du texte de Kafka renverront à l'édition suivante: Franz Kafka, Der Verschollene. Roman, in der Fassung der Handschrift, Fischer Taschenbuch Verlag, 1993 (V).

4. « Es war wirklich als gebe es keinen Heizer mehr » (V 42). 
Or, dans les deux cas, ce qui aurait pu constituer un incident négligeable - ou du moins, un fait social inaperçu - est traité par la littérature comme une scène non seulement digne d'attention, mais de la plus haute importance : il faut une nouvelle entière pour accompagner Bartleby dans sa sortie de scène définitive, et un premier chapitre consistant pour intéresser le lecteur au sort d'un soutier anonyme qui ne réapparaîtra plus de tout le roman. Mais si la littérature a jugé nécessaire de porter témoignage de la chute et de la noyade de ces deux malheureux, elle ne le fait nullement en défense des victimes. Elle n'adopte pas, ici, le point de vue du laissé-pourcompte, et ne semble pas même se donner pour but de plaider pour lui. Face à l'indifférence de la «nuit sociale» et de la «mer» qui se referme sur Bartleby et sur le soutier ${ }^{5}$, la littérature n'oppose ni le misérabilisme, ni l'apitoiement. Ni même l'humanité, pourrait-on ajouter, car l'individuation des deux laissés-pour-compte, chez Melville comme chez Kafka, reste très discrète. Leur passé est tout juste évoqué, sans insister, assez pour que l'on voie l'homme sous le cas d'espèce, mais pas assez pour que la pitié balaie toute autre considération. Tout se passe, en somme, comme dans la célèbre confrontation de K. et du chef de la commune dans un autre roman de Kafka. Face au représentant de l'administration publique, le K. du Château opposait certes sa «personne réelle » (meine wirkliche Person) cherchant à faire valoir ses « droits » (mein Recht), mais n'usait qu'en passant de la référence aux sacrifices personnels consentis par lui : "Je vais vous énumérer quelques-unes des raisons qui me retiennent ici : les sacrifices que j'ai faits pour partir de chez moi; le long et difficile voyage; les espoirs motivés que j'ai nourris à propos de ma nomination ici ; mon manque total de ressources; l'impossibilité où je suis maintenant de retrouver dans mon pays un travail équivalent $[\ldots] »^{6}$. Son «énumération » même prive en partie ce passé de sa force de passif capable d'obliger son interlocuteur, tout en rappelant tout de même le lecteur, sinon son interlocuteur, à son devoir d'empathie. La rumeur (non vérifiée) au sujet de Bartleby, racontée in extremis par le narrateur de la nouvelle de Melville dans l'épilogue, joue un rôle similairement ambivalent: «La rumeur, donc, voulait que Bartleby eût exercé une fonction subalterne au service des Lettres au Rebut de Washington, et qu'il en eût été soudainement jeté hors par un changement administratif » ( $B$ 137) ${ }^{7}$. Et le narrateur de laisser alors libre cours à sa méditation mélancolique sur cet « homme

5. Ou face à l'anonymat des chiffres et des bilans des politiques publiques tenant le compte de l'exclusion sociale au lieu d'en tenir compte.

6. Kafka, Le Château, trad. Bernard Lortholary, Paris, GF, 1984, p. 103. «Ich werde Ihnen einiges davon aufzählen was mich hier zurückhält : die Opfer, die ich brachte, um von zuhause fortzukommen, die lange schwere Reise, die begründeten Hoffnungen, die ich mir wegen der Aufnahme hier machte, meine vollständige Vermögenslosigkeit, die Unmöglichkeit jetzt wieder eine entsprechende Arbeit zuhause zu finden » Franz Kafka, Das Schloß, in der Fassung der Handschrift, Fischer Taschenbuch Verlag, p. 93.

7. «The report was this : that Bartleby had been a subordinate clerk in the Dead Letter Office at Washington, from which he had been suddenly removed by a change in the administration $\gg(B$ 136). 
au rebut » qu'est Bartleby : «Quand je songe à cette rumeur, je puis à peine exprimer l'émotion qui s'empare de moi. Les lettres au rebut! Cela ne rendil point le son d'hommes au rebut? Imaginez un homme condamné par la nature et l'infortune à une blême désespérance; peut-on concevoir besogne mieux faite pour l'accroître que celle de manier continuellement ces lettres au rebut et de les préparer pour les flammes? $\gg^{8}$ Cette corde sensible n'est jouée qu'à l'extrême fin de la nouvelle, une fois l'épisode Bartleby refermé, et qui plus est, dans un cadre qui en relativise la portée sur le personnage concerné. Si ce passé supposé du héros augmente la culpabilité subjective de ses interlocuteurs tandis qu'il était encore en vie, ce passif diminue aussi leur culpabilité objective à présent qu'il est mort. En tout état de cause, la rumeur finalement ajoutée par Melville rend tout de même au défunt Bartleby un peu de cette humanité qui lui faisait si radicalement défaut dans la nouvelle : en humanisant à peine, juste le temps d'un doute ou d'un soupçon, son personnage si énigmatique, Melville renvoie le lecteur à ses responsabilités face à cet être humain malgré tout, et rappelle du même coup la fable à sa dimension politique fondamentale. Non seulement la narration n'accentue pas outre mesure l'enjeu humaniste au détriment de la question politique, mais elle ne poursuit pas l'objectif de capter notre commisération. Dans le cas de Bartleby, comme dans celui du soutier, comme du reste dans le cas du héros du Château, les laissés-pour-compte ne sont pas des figures aimables ou attachantes. Ce sont - chacun dans son genre - des fous, des lunatiques, des fauteurs de désordre, des ingrats à la mauvaise volonté flagrante. Si cette folie, ce désordre ou ce dysfonctionnement dit assurément quelque chose de leur détresse, la littérature n'éprouve pas d'indulgence pour les malheureux dont elle décrit l'exclusion.

En outre, le traitement narratif du problème de l'exclusion dans ces deux textes n'en simplifie pas l'enjeu social. Comme dans la discussion entre K. et le chef de la commune, qui fait droit aux répliques du collectif face aux revendications de l'individu?, l'oncle Jacob peut déclarer, sans être discrédité par la narration, que la discipline à bord d'un bateau n'est pas moins importante

8. «When I think over this rumor, hardly can I express the emotions which seize me. Dead letters! Does it not sound like dead men? Conceive a man by nature and misfortune prone to a pallid hopelessness, can any business seem more fitted to heighten it than that of continually handling these dead letters, and assorting them for the flames? » (B 136).

9. «Vous êtes accepté comme géomètre, comme vous le dites, mais malheureusement nous n'avons pas besoin de géomètre. Il n'y aurait pas pour lui le moindre travail. Les limites de nos petites exploitations sont fixées, tout est enregistré en bonne et due forme, il n'y a guère de mutations et les petits conflits de bornages se règlent entre nous. Qu'aurions-nous donc à faire d'un géomètre? » Telle est la réplique - imparable - du chef de la commune à K. dans Le Château, op. cit., p. 87. « Sie sind als Landvermesser aufgenommen, wie Sie sagen, aber, leider, wir brauchen keinen Landvermesser. Es wäre nicht die geringste Arbeit für ihn da. Die Grenzen unserer kleinen Wirtschaften sind abgesteckt, alles ist ordentlich eingetragen, Besitzwechsel kommt kaum vor und kleine Grenzstreitigkeiten regeln wir selbst. Was soll uns also ein Landvermesser? » (F. Kafka, Das Schloß, Roman, op. cit., p. 75). 
que la justice, surtout quand il est question d'un soutier chicaneur, qui n'obéit pas aux ordres et poursuit sa hiérarchie de ses tracasseries. Plus clairement encore, dans la nouvelle de Melville, l'impératif de productivité du cabinet juridique et la désorganisation patente que Bartleby lui cause, ne sont nullement sous-estimés : le lecteur est invité à les prendre à son compte. Sur le plan des arguments, l'exclusion est méritée, et le récit, dans les deux cas, en prend acte. Et pourtant, ces deux textes agissent tout autrement.

La comparaison de ces deux scènes politiques américaines doit donc être articulée davantage. Dans les deux cas, un pauvre homme singulièrement désarmé et impuissant se trouve broyé dans une relation de pouvoir. Dans la cabine du capitaine, le soutier et son avocat improvisé Karl Rossmann sont seuls face à une coalition de puissants. Et dans l'affaire Bartleby, l'inégalité du rapport de forces social est évidente, entre le patron et l'employé, le notable et le pauvre hère, l'homme installé et le sans-abri. Mais ces rapports de forces sont modulés par la puissante matrice symbolique de la démocratie libérale américaine. Certes, dans cette société de marché, l'insertion sociale passe nécessairement par l'utilité, il y a des règles du jeu auxquelles chacun doit se conformer, la vie n'y est pas gratuite et on travaille pour gagner sa place au soleil : « Ici, il ne fallait pas espérer de la pitié, et ce que Karl avait lu là-dessus concernant l'Amérique était exact » $(A 53)^{10}$. Mais la matrice démocratique libérale reste ouverte : la scène américaine n'est pas le décor d'une société figée. Le pauvre et le sans-pouvoir ne sont pas condamnés à le rester. Ainsi, dans l'Amérique de Kafka, chacun peut défendre ses droits : même un soutier anonyme peut venir déranger le commandant du bateau au moment où celui-ci doit régler des affaires de la plus haute importance. Le rapport de forces reste mobile : débouté par le caissier principal, le soutier peut s'adresser directement au commandant par-dessus la tête de sa hiérarchie. Son impudence ${ }^{11}$ est explicitement tolérée par le commandant du navire : "Mais déjà le commandant intervenait, disant: - Écoutons donc cet homme. De toute façon, je trouve de plus en plus que ce Schubal en fait un peu trop à sa tête; ce qui ne signifie pas pour autant que je me prononce en votre faveur $\gg(A 31)^{12}$. Karl Rossmann le remarque avec justesse : rien n'est joué d'avance. De même, chez Melville, un simple employé peut oser répondre « je préférerais pas » (/ would prefer not to) à un

10. «Denn auf Mitleid durfte man hier nicht hoffen und es war ganz richtig, was Karl in dieser Hinsicht über Amerika gelesen hatte » (V 43).

11. «Combien de fois on vous a rappelé gentiment que, Schubal étant votre supérieur immédiat, c'est avec lui seul que vous devez régler vos affaires, en subordonné que vous êtes! Et voilà maintenant que vous vous présentez même quand le commandant est là, sans avoir honte de l'importuner, même lui [...] »A 30. «Wie oft hat man Ihnen im Guten gesagt, das Schubal Ihr unmittelbarer Vorgesetzter ist, mit dem allein Sie sich als sein Untergebener abzufinden haben! Und jetzt kommen Sie gar noch her, wenn der Herr Kapitän da ist, schämen sich nicht, sogar ihn zu belästigen... » (V 22).

12. «Aber da war auch schon der Kapitän da, welcher sagte : Hören wir den Mann doch einmal an. Der Schubal wird mir so wie so mit der Zeit viel zu selbstständig, womit ich aber nichts zu Ihren Gunsten gesagt haben will » (V 21). 
ordre de son patron : l'affaire Bartleby atteste un espace de liberté à l'intérieur du champ social qui n'est pas à sous-estimer. Plus largement, même une fois consommée la rupture avec le travail de scribe, en Amérique il reste encore toujours d'autres possibilités à envisager : c'est le sens de ce truculent dialogue de conseil en reconversion professionnelle dans lequel l'avocat suggère successivement à son ancien scribe squatteur différentes alternatives professionnelles, dont le décalage flagrant avec le caractère de Bartleby crée une tension comique ${ }^{13}$. On retrouve là un trait symbolique fort de la matrice démocratique : en démocratie, chacun peut théoriquement trouver sa place, il n'y a pas d'hommes en trop, mais chaque individu est comme en excès par rapport à lui-même. Un personnage démocratique déborde toujours ce qu'on veut qu'il soit ${ }^{14}$. À Bartleby, aucun métier ne convient parfaitement. Quant au soutier, sa logorrhée débordant de colère et d'émotion témoigne aussi de son refus d'être cantonné dans une place et un rôle : il ne veut pas être ce que Schubal, son supérieur, lui assigne d'être, il est tout autre chose que ce que quiconque prétendrait dire de lui. À la différence du K. du Château, qui revendique une utilité sociale pour laquelle le collectif n'a pas besoin de lui, Bartleby et le soutier ne veulent plus occuper le rôle auquel on les assigne. Bartleby refuse de jouer le jeu, et même de s'en expliquer. Le soutier, lui, ne sait pas se faire valoir, ni même se défendre ou se prendre en charge; dans la microsociété du navire, il se désigne aux regards comme un homme qui ne sait pas jouer le jeu social. Contrastant avec la foule de Wall Street, ou celle d'un navire arrivé à son port de destination, Bartleby et le soutier sont deux «épaves au milieu de l'Atlantique » $\left(B\right.$ 87) ${ }^{15}$ que la narration saisit au cœur de leur solitude, dans la cabine du soutier, qui sert de refuge à Karl dans le labyrinthe trépidant du navire, et dans le silence dominical d'un quartier des affaires dépeuplé.

Or, dans les deux textes, la présence de ces deux laissés-pour-compte soulève la même question : qu'en faire? Puisqu'ils sont incapables de faire quelque chose d'eux-mêmes, que sera-t-il fait d'eux? La question est directement formulée par le narrateur avocat à propos de son ancien scribe : «Allons, c'est une nécessité, de deux choses l'une: ou bien vous ferez

13. «Aimeriez-vous à être commis aux écritures dans une épicerie? [...] Aimeriez-vous à tenir un bar? [...] Aimeriez-vous à courir le pays en encaissant des factures pour le compte de marchands? [...] Vous plait-il alors d'accompagner en Europe quelque jeune homme de bonne famille qui profiterait des avantages de votre conversation? » (B 119121). « Would you like a clerkship in a dry-goods store? [...] How would a bartender's business suit you? [...] Well, then, would you like to travel through the country collecting bills for the merchants? [...] How, then, would going as a companion to Europe, to entertain some young gentleman with your conversation - how would that suit you? » (B 118-120).

14. Voir les analyses de Bakhtine, dans Esthétique et Théorie du Roman, Paris, Gallimard, coll. «Bibliothèque des Idées », 1978, («Récit épique et roman ») sur le personnage de roman comme identité toujours in-incarnable, inadéquate à elle-même, en excédent d'humanité - définition démocratique du personnage de roman.

15. «A bit of wreck in the mid-Atlantic $(B 86)$. 
quelque chose de vous-même, ou bien on fera quelque chose à votre sujet » $(B \text { 119 })^{16}$. Mais c'est également en ces termes - politiques - que doit être entendue la question inquiète de Karl : «que va-t-on faire du soutier? » $(A$ 46) ${ }^{17}$ Que faire d'un tel homme en démocratie? De cette question commune, que l'existence de ces hommes - leur présence en travers du chemin, comme celle du K. du Château uber in weg - pose au collectif, deux individus héritent à leur compte personnel : Karl Rossmann et l'avocat narrateur. Le hasard de la rencontre, matière de l'intrigue fictionnelle, fournit à la question politique la médiation du rapport éthique : «Que faire d'un tel homme? » se transforme en «que faire face à un tel homme?». Que se joue-t-il donc dans cette articulation du politique et de l'éthique?

Chacun à sa manière, et pour des raisons différentes, Karl Rossmann et l'avocat narrateur de la nouvelle de Melville se sentent personnellement interpellés par la détresse solitaire de l'homme qu'ils viennent de rencontrer : s'ils ne sauraient en être tenus pour responsables, ils acceptent pourtant d'avoir à en répondre. La passion de Karl pour le soutier, facilement décryptée par l'oncle Jacob, part d'un simple réflexe de sympathie: en entendant le soutier se plaindre de l'injustice qui le frappe, Karl Rossmann éprouve une indignation spontanée à laquelle il s'abandonne entièrement, si bien que, quelques minutes après avoir fait sa connaissance, Karl s'improvise défenseur de la cause du soutier. Non seulement il s'implique, mais il s'offre tout entier au service du soutier, se met lui-même en gage, tendant son passeport et renonçant à chercher sa valise égarée, prêt même à se bagarrer aux côtés du soutier si l'altercation tourne mal. Attitude non sans noblesse, mais infantile, jugera son oncle, tant il est vrai que Karl agit comme un travailleur social qui ne saurait pas maintenir entre lui et celui qu'il aide la distance nécessaire à sa propre survie. Il en va tout autrement de la réaction de l'avocat narrateur de la nouvelle de Melville. À l'exception des tout premiers moments de confrontation avec l'énigmatique formule de Bartleby, l'avocat se protège soigneusement de toute réaction spontanée. Il réfléchit, temporise, prend la mesure des enjeux qui se présentent à lui. C'est à l'issue d'une délibération prudente qu'il choisit d'être patient avec Bartleby, de lui tendre la main, et, plus tard dans la nouvelle, d'assumer les rôles de médiateur face à la collectivité hostile, et même d'ange gardien quand Bartleby est en prison. Si Karl vole au secours du soutier sans arrière-pensées, l'avocat de Melville, lui, se rend bienveillant après maints efforts sur soi-même pour contenir son exaspération et sa violence. Sa charité - puisque c'est le terme qu'il emploie - n'a rien de spontané, c'est un carcan qu'il s'impose à luimême pour s'empêcher de commettre des actes graves sous le coup de la colère. En homme très cultivé, doté en particulier d'une culture biblique très maîtrisée, il déchiffre d'emblée l'enjeu de cette rencontre, y reconnaît

16. «Now one of two things must take place. Either you must do something, or something must be done to you » (B 119).

17. «Was wird jetzt dem Heizer geschehn? » (V 37). 
l'épreuve du Bon Samaritain et sa définition du prochain, lié par «le lien d'une humanité commune » $(B \text { 69 })^{18}$, et décide d'agir - ne serait-ce que dans son propre intérêt - conformément à l'éthique biblique. Contrairement à Karl qui, voyant un homme blessé sur le bas-côté de sa route, met pied à terre, renonce à poursuivre son propre trajet et donne tout de sa personne pour lui venir en aide, l'avocat de Melville, lui, reconnaît immédiatement dans son homme blessé à lui l'épreuve biblique de la parabole christique. Loin de toute pitié spontanée, sa culture et sa morale servent de médiation entre lui et le spectacle du monde. Il se demande ce qu'il doit faire, met pour lui-même en scène sa propre perplexité morale («Que vais-je faire? me demandai-je alors en boutonnant ma jaquette jusqu'au dernier bouton? Que vais-je faire? Que dois-je faire? Qu'est-ce que ma conscience me dicte au sujet de cet homme? ${ }^{19}$ ) et y répond en toute connaissance de cause. Et, lucide sur sa propre démarche, se réjouit que cela marche : «C'est l'une des moindres vertus de la charité qu'elle opère souvent comme un grand principe de sagesse et de prudence; elle est pour qui la possède une excellente sauvegarde. [...] Leur seul intérêt personnel, à défaut d'un motif meilleur, devrait donc inciter tous les hommes, et particulièrement les tempéraments emportés, à la charité et à la philanthropie » $(B 101)^{20}$. La charité de ce Bon Samaritain d'adoption consiste à tolérer Bartleby dans son espace, à lui permettre une présence gratuite, soustraite au jeu du marché, sans prise en compte de son utilité : au nom de leur «humanité commune », accepter dans ses bureaux un homme qui n'a rien à y faire, qui n'y sert à rien, mais ne coûte rien non plus (du moins le croit-il au début) suppose aussi d'accepter pour lui de braver l'incompréhension des autres.

Les deux scénarios fictionnels butent alors sur la même question en filigrane : jusqu'où peut-on donner de soi pour tenter de sauver un semblable qui ne nous est rien - rien d'autre qu'un « prochain »? Face à leur blessé de rencontre, nos deux Samaritains donnent d'eux-mêmes très largement, jusqu'à se mettre en risque - puis se retirent. À un moment donné, la main tendue est reprise, le protégé est abandonné à son triste sort.

Cette main lâchée, le texte de Kafka la met en scène dans le cadre d'un roman de formation. Alors que Karl Rossmann s'est montré prêt à sortir du terrain de jeu social pour tenter d'y réinsérer le soutier, la question du sénateur Jacob le rappelle à lui-même et à sa propre place. «Comment vous appelez-vous? » Cette question à laquelle Karl ne prête aucune attention, c'est le commandant lui-même, arbitre du conflit du soutier, qui l'oblige à y répondre. Karl s'en acquitte d'abord sans comprendre qu'il est vraiment

18. «the bond of a common humanity » (B 68).

19. «What shall I do? I now said to myself, buttoning up my coat to the last button. What shall I do ? What ought I to do? What does conscience say I should do with this man » (B 108-109).

20. «Aside from higher considerations, charity often operates as a vastly wise and prudent principle - a great safegard to its possessor. [...] Mere self-interest, then, if no better motive can be enlisted, should, especially with high-tempered men, prompt all beings to charity and philanthropy » (B 100). 
concerné, et ayant appris que l'homme à la badine est son oncle, s'efforce, «sans émotion aucune », de "prévoir les conséquences qu'aurait pour le soutier cet événement nouveau » dont «pour l'instant, rien n'indiquait que Schubal pût tirer avantage ». Le commandant le remet alors dans le bon chemin, exactement comme on ferait avec un enfant qui ne comprend pas la situation : «C'est le Sénateur Edward Jakob, qui vient de vous révéler qu'il est votre oncle. C'est désormais, contrairement sans doute à tout ce que vous pouviez jusqu'à présent espérer, une carrière éblouissante qui vous attend. Essayez de comprendre cela, autant qu'il est possible sur le moment, et ressaisissez-vous » $\left(A\right.$ 39) ${ }^{21}$. Le nouveau rang de Karl, fait brusquement basculer l'affaire du soutier à l'arrière-plan : pire encore, le soutier n'est plus que l'instrument des retrouvailles de Karl avec son oncle, qui raconte joyeusement comment «l'insupportable bavardage du soutier » lui a donné l'occasion de «tirer son calepin dans le seul but de se distraire » et de « confronter avec la figure de Karl les observations de la cuisinière » qui l'avait prévenu de l'arrivée de son neveu. C'en est assez, aux yeux du sénateur, pour justifier qu'on abandonne enfin le soutier à son sort sans plus s'en soucier. Première étape d'un roman d'initiation: le personnage principal doit se résoudre à quitter le souci moral infantile pour entrer dans la sphère de la politique, où les rapports de pouvoir, les règles de vie commune et les considérations d'utilité l'emportent. Le franchissement de ce seuil initiatique de sortie de l'enfance politique est ainsi commenté par l'oncle Jakob : «Tu te sentais abandonné et tu as trouvé le soutier, alors maintenant tu lui es reconnaissant, c'est tout à fait louable. Mais ne pousse pas les choses trop loin, ne serait-ce qu'à cause de moi, et conçois la situation qui est désormais la tienne » $\left(A\right.$ 49) ${ }^{22}$

La nouvelle Bartleby n'est pas bâtie sur un schéma similaire de maturation politique. Dès le départ, le narrateur se présente avec insistance comme un homme d'un certain âge, parfaitement inséré socialement, sérieux et raisonnable: sa charité envers Bartleby, on l'a vu, a été décidée après mûre réflexion. Or, deux raisons conjointes déclenchent pourtant l'abandon de Bartleby. Il y a d'abord le coût social trop élevé de cette charité, que le narrateur croyait gratuite, mais qui risque de lui coûter sa réputation et sa place dans le jeu social. La charité n'a qu'un temps. Un homme à la mer? Qui voudrait lui tendre la main, quand les vagues sont déchaînées, la compétition impitoyable, et qu'on risque de basculer soi-même par-dessus bord, entraîné par le poids? Craignant que sa pitié ne lui coûte sa vie (sociale), l'avocat décide de couper tout lien avec Bartleby. Il se dédouane de toute responsabilité, tente de se rendre indifférent en se déclarant non concerné : «Je

21. «Es ist der Staatsrat Edward Jakob, der sich Ihnen als Ihr Onkel zu erkennen gegeben hat. Es erwartet Sie nunmehr, doch wohl ganz gegen Ihre bisherigen Erwartungen eine glänzende Laufbahn. Versuchen Sie das einzusehn, so gut es im ersten Augenblick geht und fassen Sie sich » (V 30).

22. «Du hast Dich verlassen gefühlt, da hast Du den Heizer gefunden und bist inm jetzt dankbar, das ist ja ganz löblich. Treibe das aber, schon mir zuliebe, nicht zu weit und lerne Deine Stellung begreifen » (V 40). 
regrette beaucoup, monsieur, répondis-je avec une feinte tranquillité, mais aussi avec un tremblement intérieur; en vérité l'homme auquel vous faites allusion ne m'est rien, il n'est ni mon parent ni mon employé, et vous ne sauriez me rendre responsable de lui » $(B \text { 115 })^{23}$. C'est encore ici la culture biblique de l'avocat narrateur qui fonctionne comme intertexte: toute la parabole du Bon Samaritain vise à définir le prochain en dehors de tous les liens d'imputation et de responsabilité qui structurent le tissu social ${ }^{24}$. La charité n'est pas une solution politique? La Bible ne disait pas autre chose, justement, et notre homme le sait. Le niveau auquel se situe la charité et celui de la politique sont disjoints. La première est bien incapable de résoudre les problèmes de la seconde, mais elle n'en a nullement la vocation.

Mais il y a une autre raison, plus profonde, qui entraîne l'avocat narrateur à lâcher la main de Bartleby. Cette raison, c'est l'impasse de la pitié. L'avocat raconte qu'il en prend conscience le dimanche où, passé au bureau à l'improviste, il surprend Bartleby sur place et comprend qu'il n'a nulle part ailleurs où vivre - pour employer délibérément une expression aux connotations dostoïevskiennes: «Mes émotions premières avaient été de pure mélancolie et de la plus sincère pitié; mais à mesure que la détresse de Bartleby prenait dans mon imagination des proportions de plus en plus grandes, cette même mélancolie se muait en frayeur, cette pitié en répulsion. Tant il est vrai et terrible à la fois que, jusqu'à un certain point, l'idée ou la vue du malheur mobilise nos meilleurs sentiments, mais que, dans certains cas particuliers, au-delà de ce point elle ne les commande plus » (B 73) ${ }^{25}$. Il faut prendre au sérieux ce discours sur l'impuissance de la pitié, sans l'imputer trop vite à l'écriture thérapeutique de la culpabilité avec laquelle le narrateur se débat tout au long de sa confession. L'aporie de la pitié tiendrait au caractère incommunicable du malheur extrême : il n'y a plus rien en commun, plus rien qui puisse être partagé, transmis et allégé d'un être à un autre. Celui qui mérite le plus la pitié, c'est en somme celui pour lequel on ne peut rien, celui pour lequel personne ne peut rien, celui qui est trop fou, trop malheureux, trop perdu pour être aidé, celui qui est déjà au-delà de toute aide humaine possible... C'est peut-être dans cette perspective que l'on doit comprendre la scène, à la fois désopilante et insupportable, du dialogue sur les perspectives de réinsertion professionnelle de Bartleby, quand ce dernier balaie systématiquement toutes les suggestions

23. « I am very sorry, sir, said I, with assumed tranquillity, but in an inward tremor, but, really, the man you allude to is nothing to $\mathrm{me}-$ he is no relation or apprentice of mine, that you should hold me responsible for him » (B 114).

24. Voir l'analyse que fait Luc Boltanski de la parabole du Bon Samaritain comme support d'une conceptualisation juridique du devoir d'assistance à personne en danger, dans La Souffrance à distance. Morale humanitaire, médias et politique, Paris, éd. Métailié, 1993, p. 21-26.

25. «My first emotions had been those of pure melancholy and sincerest pity; but just in proportion as the forlornness of Bartleby grew and grew to my imagination, did that same melancholy merge into fear, that pity into repulsion. So true it is, and so terrible too, that up to a certain point the thought or sight of misery enlists our best affections; but, in certain special cases, beyond that point it does not » (B 72). 
que lui fait l'avocat, tout en prétendant n'être « pas difficile »: Bartleby refusant toute aide, c'est Bartleby au-delà de toute aide possible. Trop fou pour être sauvé. Répugnant de détresse.

Il y avait, du reste, déjà quelque chose de ce découragement, de cette répulsion troublante et inavouable, dans la réaction à fleur de peau de Karl Rossmann face au soutier apathique, comme résigné à sa propre défaite : «- Mais pourquoi ne dis-tu rien? lui dit-il. Pourquoi te laisses-tu faire sans réagir? » (A 48) «- Mais il faut que tu te défendes, que tu dises oui ou non, sinon les gens ne soupçonneront pas la vérité » $(A 49)^{26}$. Entouré d'ennemis, ne sachant où poser les yeux, sinon sur Karl, le soutier ne sait pas plaider sa cause, ne sait même pas l'exposer en termes intelligibles. Le «pauvre homme en butte aux grands $»(A 31)^{27}$ se répand en plaintes et en récriminations désordonnées, dont «il ne ressortait rien de précis», et ce « tourbillon confus » impatiente tout le monde; comme le remarque Karl, le soutier va même jusqu'à traiter le commandant comme « un instrument dont on pouvait jouer à tort et à travers », «même si c'était sous le coup d'une révolte qui ne connaissait plus de limites $\gg(A 33)^{28}$. Mais comment le soutier feraitil autrement? «Ses yeux étaient tout embués par les larmes de son honneur blessé, de ses souvenirs terribles, de son extrême détresse présente, et il n'était déjà plus capable de bien distinguer Karl. Comment aurait-on voulu - Karl s'en rendit compte en silence, face à cet homme à présent silencieux - , comment aurait-on voulu que tout d'un coup, maintenant, il changeât aussi de discours, alors qu'il avait l'impression d'avoir déjà exposé tout ce qu'il y avait à dire sans éveiller le moindre écho, et en même temps de n'avoir rien dit, sans pouvoir pourtant exiger de ce monsieur d'écouter tout une nouvelle fois encore? $\gg(A 34)^{29}$. L'incapacité du soutier à se défendre fait perdre toute légitimité à sa cause. Sa détresse même le rend fou, et cette folie lui ôte ses droits. Sa souffrance le disqualifie socialement, le fait apparaître comme irrécupérable, définitivement inadapté.

Avec Bartleby, comme avec le soutier, en apparence seulement moins affecté, la fiction attire l'attention sur un fait que l'économie connaît statistiquement, mais que la politique ne peut reconnaître, et encore moins traiter :

26. «Warum sagst Du denn nichts? fragte er. Warum läßt Du Dir alles gefallen? [...] Du mußt Dich aber zur Wehr setzen, ja und nein sagen, sonst haben ja die Leute keine Ahnung von der Wahrheit » (V 39-40).

27. « der unter die Großen gestellten armen Mannes einen Teil mitfühlte » (V 22).

28. «aber schließlich war er kein Instrument, das man in Grund und Boden spielen konnte - und gerade so behandelte ihn der Heizer, allerdings aus seinem grenzenlos empörten Inneren heraus » ( $V$ 23).

29. «mit seinen Augen, die ganz von Tränen, der beleidigten Mannesehre, der schrecklichen Erinnerungen, der äußersten gegenwärtigen Not verdeckt waren, konnte er Karl schon nicht einmal gut mehr erkennen. Wie sollte er auch jetzt, Karl sah das schweigend vor dem jetzt Schweigenden wohl ein, wie sollte er auch jetzt plötzlich seine Redeweise ändern, da es ihm doch schien, als hätte er alles was zu sagen war ohne die geringste Anerkennung schon vorgebracht und als habbe er andererseits noch gar nichts gesagt und könne doch den Herren jetzt nich zumuten, noch alles anzuhören » (V 24). 
il y aurait donc, dans les sociétés démocratiques modernes, des inadaptés, des « in-insérables», des hommes que leur malheur même rend indéfendables, que leur détresse même condamne à la folie et au rebut? Ainsi du K. du Château, cet homme inadapté et dysfonctionnel, qui ne sait pas jouer le jeu du Château et que tous les habitants du village traitent comme un enfant, un fou ou un paria, ce K. qui, au nom même de sa détresse, harcèle Klamm au point de l'attendre dans son traîneau, démesure qui condamne toute chance d'échapper à un isolement presque sanitaire... Ou celui de Bartleby qui, après le déménagement de son ancien patron, s'obstine à occuper l'escalier de l'immeuble, empêchant tout le monde de travailler par sa seule présence intempestive. Folie, détresse? Cela revient au même, dans une logique du pire qui exclut toute pitié : plus il est isolé, plus il est malheureux, plus il est malheureux, plus il est fou, plus il est fou, plus on doit l'isoler. Il y a, en effet, dans tout processus d'exclusion, quelque chose du rouage maléfique que décrit Hugo dans les Misérables - une logique de damnation.

Reste à savoir comment l'on traite ces parias, une fois reconnus et identifiés comme tels. À la question de savoir que faire de ces laissés-pourcompte, la fiction de Kafka et de Melville répond d'abord de même : rien. On ne fait rien, et c'est la seule manière de réagir dans le respect formel des droits des personnes, tout en tentant l'ultime moyen de réduire leur résistance. Lorsque K. campe dans le traîneau de Klamm, bien décidé à l'attendre le temps qu'il faudra, Klamm fait dételer. Le cocher rentre les chevaux, et $K$. se retrouve tout seul, dans la cour enneigée de l'auberge : maître des lieux, libre et invulnérable, «plus libre que jamais », mais terriblement seul, car « il n'y avait rien de plus dépourvu de sens, rien de plus désespéré que cette liberté, cette attente, cette invulnérabilité ${ }^{30}$. La liberté du paria dit quelque chose de la liberté tout court : la liberté, en démocratie, ne saurait se définir seulement par l'émancipation de l'individu, soumis à aucune contrainte autre que celle de la loi, libre de ne pas être inquiété. Sans la possibilité de contracter des liens avec les autres, cette liberté démocratique est vaine : K., brisé, quitte les lieux. La même expérience, inoffensive et

30. «K. eut le sentiment qu'on avait coupé toute liaison avec lui, qu'il était désormais certes plus libre que jamais et qu'en ce lieu naguère interdit il pouvait attendre aussi longtemps qu'il voulait, qu'il avait conquis cette liberté de haute lutte comme personne d'autre ou presque et que nul n'avait le droit de le toucher ou de le chasser, ni même de lui adresser la parole, mais (et cette conviction était au moins aussi forte) qu'il n'y avait rien de plus dépourvu de sens, rien de plus désespéré que cette liberté, cette attente, cette invulnérabilité. » Le Château, op. cit., p. 140. « da schien es K., als habe man nun alle Verbindung mit inm abgebrochen und als sei er nun freilich freier als jemals und könne hier auf dem ihm sonst verbotenen Ort warten solange er wolle und habe sich diese Freiheit erkämpft wie kaum ein anderer es könnte und niemand dürfe inn anrühren oder vertreiben, ja kaum ansprechen, aber - diese Überzeugung war zumindest ebenso stark - als gäbe es gleichzeitig nichts Sinnloseres, nichts Verzweifelteres als diese Freiheit, dieses Warten, diese Unverletzlichkeit. (Das Schloß, op. cit. p. 132-133). 
terrifiante à la fois, est menée en deux temps dans la nouvelle de Melville. D'abord, l'avocat fait comme si Bartleby n'était pas là : Bartleby consent à sa transparence. Puis il l'abandonne sur place et quitte lui-même les lieux, tournant sa liberté en dérision. Et Bartleby y consent encore. Bartleby pousse plus loin que K. l'expérimentation sur les limites de la liberté en démocratie, obligeant la fiction à affronter le problème politique dans toute sa radicalité. Il y a des hommes qui ne peuvent occuper aucune place, jouer aucun rôle, remplir aucune fonction, dans le domaine social, et qui prétendent tout de même s'y maintenir, car telle est l'humaine condition. Il n'y a pas d'homme, seul : seul, il n'y a plus d'homme. Bartleby, sur l'escalier, s'accroche au domaine public comme à un «droit de l'homme ». Si le lien social est peut-être un droit de l'homme démocratique, Bartleby en fournit une dernière preuve, sur sa vie, en refusant l'ultime solution qui peut encore le préserver de l'intervention de la force publique - l'hébergement au domicile de son ancien patron, que ce dernier lui propose en désespoir de cause. Il n'y a pas de solution privée : Bartleby maintient son problème dans le domaine public. Façon de dire aussi que l'enjeu politique de l'homme au rebut ne saurait se résoudre au niveau éthique.

Face à ces laissés-pour-compte, la littérature aura tenté une médiation. En modulant le problème sociopolitique en question éthique (via le rapport de face à face), la littérature se sera efforcée de remettre dans le jeu ces marginaux, de prendre en compte ces incomptés. Parce qu'un jour, quelqu'un s'est saisi pour lui-même de la question posée au collectif comme d'un problème personnel. Mais ce faisant, la littérature prend acte du fait que la démarche éthique ne saurait se substituer à la solution introuvable du problème politique. Sur le plan politique, c'est un échec absolu : il y a mort d'homme. Le rapport éthique ne résout rien : la leçon du Bon Samaritain ne vaut rien, dès lors que la parabole est prolongée jusqu'au point où elle démontre d'elle-même son impuissance : et si le Samaritain ne revient pas? Et s'il ne revient pas chercher le blessé qu'il a confié aux bons soins de l'aubergiste, qu'adviendra-t-il de l'infirme désormais incapable de subvenir à ses besoins?

Bien sûr, ces parias sont des fictions, d'abord au sens où le récit inventé joue ici un rôle crucial : sans narration pour le prendre en charge, sans narrateur pour en faire son sujet, Bartleby aurait été chassé dans l'indifférence, balayé par l'impitoyable réalité sociale - il n’y aurait pas de cas Bartleby. Surgit pourtant, par la fiction, à côté de l'indifférence pressée (du nouveau locataire des bureaux qu'occupe Bartleby, qui fait incarcérer le scribe), ou de la dérision cruelle (des matelots et filles de cuisine témoins rieurs du procès du soutier), une autre façon de faire voir le problème. La littérature ne donne pas à la politique le moyen de le résoudre, pas plus qu'elle ne s'en donne le moyen : le trou dans l'eau que fait le malheureux soutier en tombant à la mer se referme vite, et le personnage disparaît de la fiction comme s'il n'avait jamais existé. Mais l'espace d'un instant, en conscience, le laissé-pourcompte aura apparu, silhouette hâve et fantomatique, pantin gesticulant tout 
en sueur d'efforts pour se faire admettre, assez cependant pour persister comme une trace indélébile dans le sillage de cette humanité qui ne peut plus rien pour lui.

Frédérique LEICHTER-FLACK Université Paris $X$ Nanterre 